\title{
Uso de malla reabsorbible en traumatismo esplénico en ratas
}

\author{
Dina Sánchez ${ }^{1,2}$, Manuel Mendoza ${ }^{1}$, Guillermo Guardia ${ }^{1}$, David Sánchez ${ }^{1}$, \\ Sonia Pereyra ${ }^{2,3}$, Elena Vega ${ }^{2}$
}

Resumen

Objetivo: Determinar si el empleo de malla reabsorbible cohibe la hemorragia y evita la exéresis del bazo en el traumatismo abdominal. Diseño: Estudio experimental. Lugar: Servicio de Técnica Quirúrgica y Cirugía Experimental, Universidad Nacional Mayor de San Marcos, y Servicio de Cirugía Experimental, Hospital del Niño, Lima, Perú. Materiales: Malla reabsorbible (ácido poliglicólico), ratas, anestésicos, instrumentos de cirugía menor, material para fijación anatomopatológico. Intervenciones: Bajo anestesia general, se laparotomizó a la rata con incisión mediana, para exponer el bazo, al cual se produjo lesiones de $1^{\circ}, 2^{\circ}$ y $3^{\circ}$ grados; luego, se cubrió con la malla reabsorbible, fijando alrededor con catgut 3/0, presionando hasta cohibir la hemorragia; enseguida se cerró cavidad abdominal con puntos de vicryl 3/0. Se evaluó clínicamente a las 24, 48 y 72 horas posquirúrgicas, para observar la supervivencia; luego, relaparotomía al azar, a los 15 y 30 días y 3 meses, para ver presencia de adherencias y tiempo de reabsorción de la malla. Principales medidas de resultados: Supervivencia, adherencias posquirúrgicas y reabsorción de la malla. Resultados: De las 60 ratas con lesiones de $1^{\circ}, 2^{\circ}$ y $3^{\circ}$ grados, se observó una supervivencia de 97\% (58 ratas) y 3\% murieron (2 ratas: una rata con lesión de $1^{\circ}$ grado a las 24 horas por agresión y otra con lesión de $3^{\circ}$ grado a las 72 horas que a la necropsia se encontró microabscesos en omento e hígado). A los 15 y 30 días de relaparotomía, se observó presencia de adherencias intraabdominales y, a los 3 meses, reabsorción total de la malla. Conclusiones: La malla reabsorbible (ácido poliglicólico) es un material que, aparte de cohibir la hemorragia, evita la esplenectomía, conservando el bazo en casos de hemorragia por traumatismo abdominal.

Palabras clave Ácido poliglicólico; glicolatos; esplenectomía; traumatismos abdominales.

\begin{abstract}
Use of reabsorbing mesh in rats splenic trauma Abstract

Objective: To determine if use of reabsorbing mesh stops hemorrhage and avoids spleen removal in abdominal trauma. Design: Experimental study. Setting: Experimental Surgery Service, of both UNMSM and Children's Hospital. Materials: Reabsorbing mesh (polyglycolic acid), rats, anesthesia, minor surgery equipment, materials for anatomical tissues. Interventions: Under general anesthesia rats were opened by midline incision to expose the spleen, which was injured in 1st, 2nd and 3rd degrees, then covered with reabsorbing mesh, fixing with catgut 3/0 and pressing until bleeding was restrained; then

1 Departamento Académico de Cirugía, Facultad de Medicina Universidad Nacional Mayor de San Marcos. Lima, Perú.

2 Instituto de Salud del Niño. Lima, Perú.

3 Facultad de Odontología, Universidad Nacional Mayor de San Marcos. Lima, Perú.
\end{abstract}

abdominal cavity was closed with vicryl 3/0. Clinical evaluation was done at 24, 48 and 72 hours post-surgery to observe survival, then re-opened randomly at 15, 30 days and 3 months to see the presence of adhesions and mesh reabsorption. Main outcome measures: Survival, adhesions, mesh reabsorption. Results: From the 60 rats with 1st, 2nd, and 3rd degree lesions 97\% survived (58 rats) and 3\% died (2 rats: one rat with $1^{\circ}$ degree injury died at 24 hours because of aggression from another rat and another rat with $3^{\circ}$ degree injury died 72 hours post op and at necropsy showed micro abscesses in mesentery and liver). At 15 and 30 days we observed intra-abdominal adhesions, and at 3 months total reabsorption of the mesh. Conclusions: Reabsorbing mesh (polyglycolic acid) is a material that restrains bleeding and avoids spleen extraction in abdominal trauma bleeding.

Key words: Polyglycolic acid; glycolatos; splenectomy; abdominal injuries. 


\section{INTRODUCCIÓN}

El bazo es un órgano frecuentemente injuriado en un traumatismo abdominal cerrado. En las últimas décadas, la conservación esplénica es la más adecuada $\left({ }^{1}\right)$, teniendo en cuenta que el bazo es un órgano secundario fundamental del sistema inmunitario. Normalmente, funciona como fuente de células linforeticulares y a veces hematopoyéticas, que cesa antes del nacimiento. En casos de anemia, las células esplénicas se reactivan como órgano hematopoyético extramedular. Sirven también de depósito fundamental de fagocitos, por su rica vascularización y función fagocitaria $\left.{ }^{2}\right)$. Además, sirve de almacén y reserva del 30 a $40 \%$ de la masa total de plaquetas del organismo. Como órgano del sistema circulatorio, tiene funciones fundamentales, como el de eliminar elementos no deseados de la sangre, entre ellos la mitad de eritrocitos obsoletos, hematíes dañados y bacterias.

De los 1/120 hematíes que se destruyen en el sistema del retículo endotelial, 'selecciona' eritrocitos sin destruirlos y extrae inclusiones, como los gránulos sideróticos, cuerpos de Heinz y cuerpos de Howell-Joley $\left({ }^{3}\right)$.

Hasta los años 80 y 90, era común extirpar el bazo en casos de traumatismo abdominal y anemia hemolítica, porque se desconocía su importancia; se creía que solo cumplía su función en la etapa intrauterina y cesaba al nacimiento $\left({ }^{4}\right)$. Actualmente, se le trata de conservar.

El pionero de esta conservación es Dretzka quien, en 1930, realizó la primera esplenorrafia $\left(^{5}\right)$ y Morgenstein la primera esplenectomía parcial. En China, Chu Kin Qui, en 1992, publicó el uso de malla reabsorbible en traumatismo esplénico, para evitar la esplenectomía. Y nosotros hace más de 10 años en la Universidad Nacional Mayor de San Marcos hicimos un experimento lesionando el bazo de 10 perros y envolviéndolos con gasa por un periodo de 24 horas, obteniendo la hemostasia del $100 \%$ de casos.

Hace tres años realizamos una investigación similar en 20 ratas usando gorotex, con el mismo diseño obtuvimos una supervivencia del $90 \%$.
Si bien es cierto que las rafias y las esplenectomías parciales son comunes en traumatismos de bazo, aún se realiza esplenectomías totales para evitar muerte por shock hemorrágico $\left({ }^{6}\right)$.

El objetivo principal del presente trabajo es demostrar la efectividad de la malla reabsorbible en el traumatismo esplénico, cohibiendo la hemorragia y evitando la esplenectomía.

\section{MÉTODOS}

El presente es un estudio experimental realizado en los servicios Técnica Quirúrgica y Cirugía Experimental, de la Universidad Nacional Mayor de San Marcos y del Hospital del Niño. La unidad de investigación fue el bazo lesionado de las ratas. El material biológico utilizado fue la malla reabsorbible (ácido poliglicólico). El experimento consistió en usar 60 ratas, de 250 a 300 g cada una, de raza albina, a quienes al azar se les dividió en 4 grupos -grupo I control (10 ratas), grupo II con lesiones de $1^{\circ}$ grado (10ratas), grupo III con lesiones de $2^{\circ}$ grado (20 ratas) y grupo IV con lesiones de $3^{\circ}$ grado (20 ratas)-, a quienes se les laparatomizó con incisión mediana, bajo anestesia general (ketamina 0,03, xilocaína y atropina, vía subcutánea). Luego de ingresar a cavidad y ubicar el bazo, se produjo lesiones con bisturí, de $1^{\circ}$, $2^{\circ}$ y $3^{\circ}$ grados. Enseguida, se les envolvió con la malla reabsorbible, produciéndoles hemostasia por compresión. Una vez concluida la intervención quirúrgica, se cerró la cavidad abdominal con puntos totales y vicryl $3 / 0$ y se les aplicó a cada una antibióticos, analgésicos y dextrosa al $5 \%$, por vía subcutánea (Tabla 1 ).

Tabla 1. Grupos, grado de lesión y tiempo de muerte por hemorragia.

\begin{tabular}{cccccc}
\hline Grupo & $\mathrm{N}^{\circ}$ de ratas & $\begin{array}{c}\text { Grado de } \\
\text { lesión del bazo }\end{array}$ & $24 \mathrm{~h}$ & $36 \mathrm{~h}$ & $72 \mathrm{~h}$ \\
\hline I control & 10 & - & - & - & - \\
II & 10 & $1^{\circ}$ & No & No & No \\
III & 20 & $2^{\circ}$ & $\mathrm{Si}$ & No & No \\
IV & 20 & $3^{\circ}$ & No & No & Si \\
Total & 60 & - & - & - & 1 \\
\hline
\end{tabular}




\section{RESULTADOS}

De las 60 ratas en estudio, las lesiones de $1^{\circ}$ grado producidas al bazo de las 10 ratas del grupo II y envueltas con malla reabsorbible mostró $100 \%$ de hemostasia, con supervivencia de $100 \%$. De las 20 ratas del grupo III con lesiones de $2^{\circ}$ grado, hubo supervivencia de $95 \%$ (19 ratas); 1 rata murió por agresión de otra rata a las 24 horas. De las 20 ratas del grupo IV, con lesiones de $3^{\circ}$ grado, 1 rata murió (5\%) a las 72 horas y a la relaparotomía se observó presencia de microabscesos y adherencias intraabdominales, con una supervivencia de $95 \%$ (19 ratas) (Tabla 2).

Tabla 2. Grado de lesión y supervivencia.

\begin{tabular}{cccccc}
\hline Grupo & $\begin{array}{c}\mathrm{N}^{\circ} \text { de } \\
\text { ratas }\end{array}$ & Grado & \multicolumn{2}{c}{ Supervivencia } & Hemorragia \\
& de & Sí & No & \\
\hline I (control) & 10 & Ninguno & Todas & & - \\
II & 10 & $1^{\circ}$ & $10(100 \%)$ & - & No \\
III & 20 & $2^{\circ}$ & $19(95 \%)$ & $1(5 \%)$ & No \\
IV & 20 & $3^{\circ}$ & $19(95 \%)$ & $1(5 \%)$ & No \\
Total & $60(100 \%)$ & - & $58(97 \%)$ & $2(3 \%)$ & - \\
\hline
\end{tabular}

En los estudios anatomopatológicos se puede observar los grados de evolución del bazo y de la malla cubriendo la zona lesionada hasta observar una coalescencia entre la malla y el bazo (Figuras 1 a 5$)$.

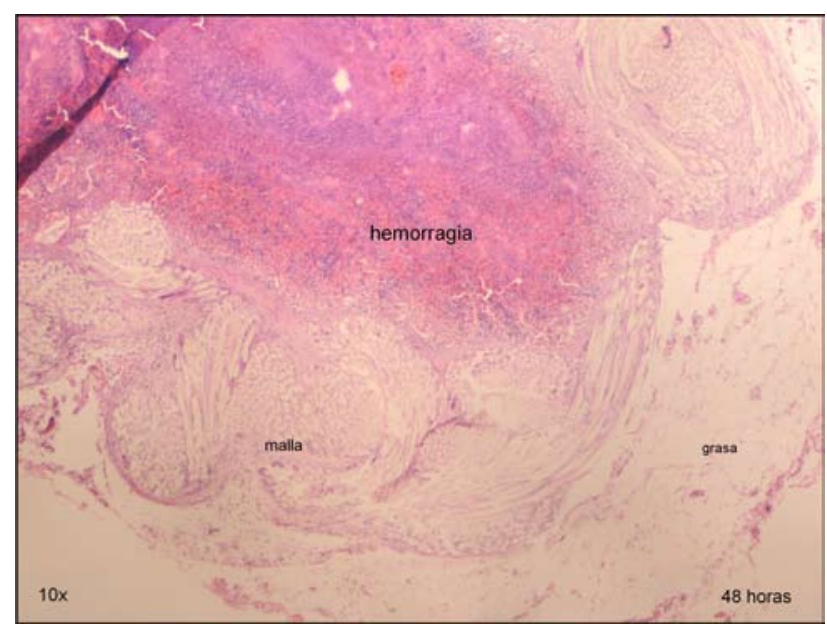

Figura 2. Amplia hemorragia en lesión de $3^{\circ}$ grado y cubierta de malla.

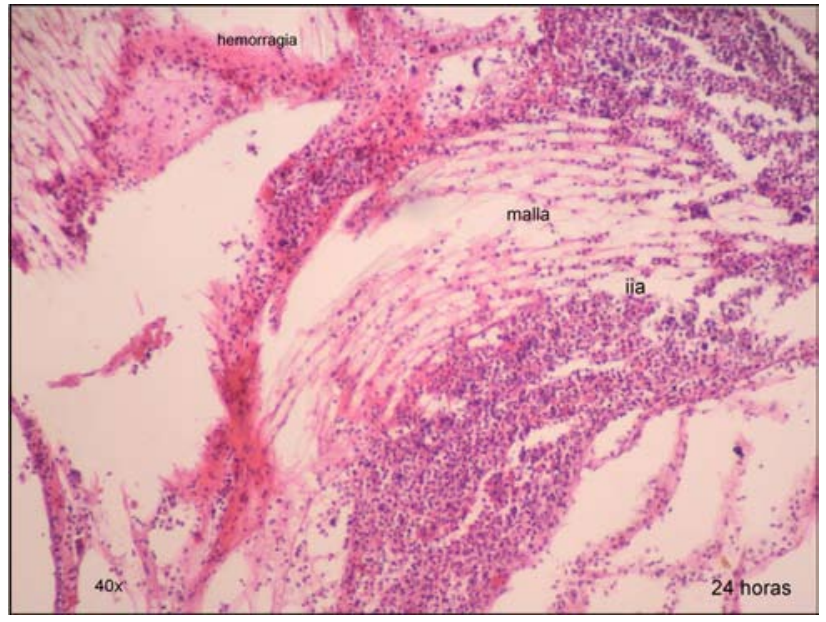

Figura 1. Corte histológico del bazo a las 24 horas, muestra hemorragia en la lesión de $2^{\circ}$ grado.

\section{DISCUSIÓN}

El tratamiento del traumatismo esplénico ha variado significativamente, por el mayor conocimiento de la fisiología del bazo. La infección postesplenectomía ha conducido a replantear la indicación quirúrgica, ante diversas lesiones traumáticas. Los pacientes pediátricos presentan en mayor número los riesgos postesplenectomía relacionados con las infecciones, los cuales son más graves cuanto menor es la edad del niño $\left({ }^{7}\right)$, indicándose técnicas de preservación esplénica $\left.{ }^{(}\right)$, resecciones

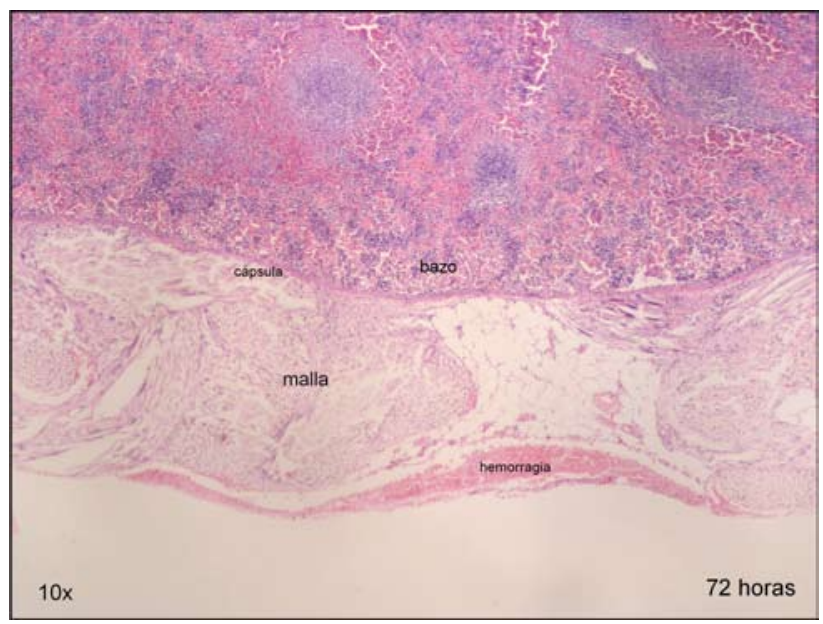

Figura 3. Presencia de macrófagos. 


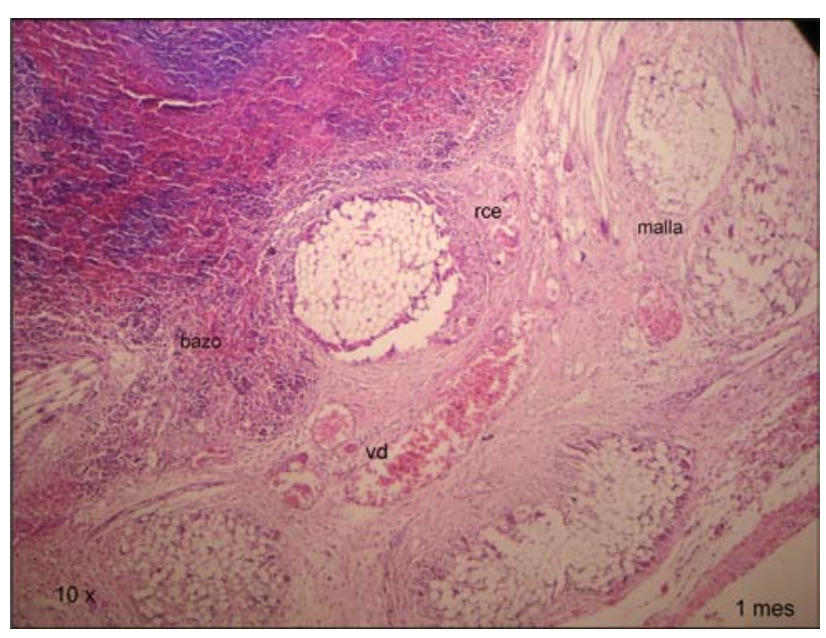

Figura 4. Reacción cicatricial en zona lesionada.

segmentarias, esplenorrafias, encestes con mallas reabsorbibles, taponamientos con fibrina, esplenectomías con autoinjerto. La capacidad de nutrirse por imbibición hace posible esta conducta, a pesar de que por el momento no se la considera una alternativa eficaz para prevenir infecciones. Para traumatismo grave, se utiliza mallas, envolviendo el bazo y con resultados alentadores $\left(^{8}\right)$.

El tratamiento conservador es más efectivo en niños, ya que con la edad aparecen cambios en la arquitectura y vascularización del bazo.

La frecuencia de traumatismo abdominal lamentablemente ha aumentado, a expensas de los accidentes de tránsito y al imprudente uso de armas de fuego.

En el pasado, en casos de traumatismo de bazo era natural extirparlo, para evitar la muerte por shock hemorrágico, aunque igual se morían los sobrevivientes por sepsis. En la actualidad, la actitud quirúrgica de todo cirujano es conservadora. Han pasado algunos años desde que los trasplantes de órganos eran una forma de preservar la función de los tejidos y por ende, la vida del ser humano.

En nuestro medio, se ha hecho común preservar el bazo a través de rafias y resecciones segmentarias. Con los resultados del estudio de la malla

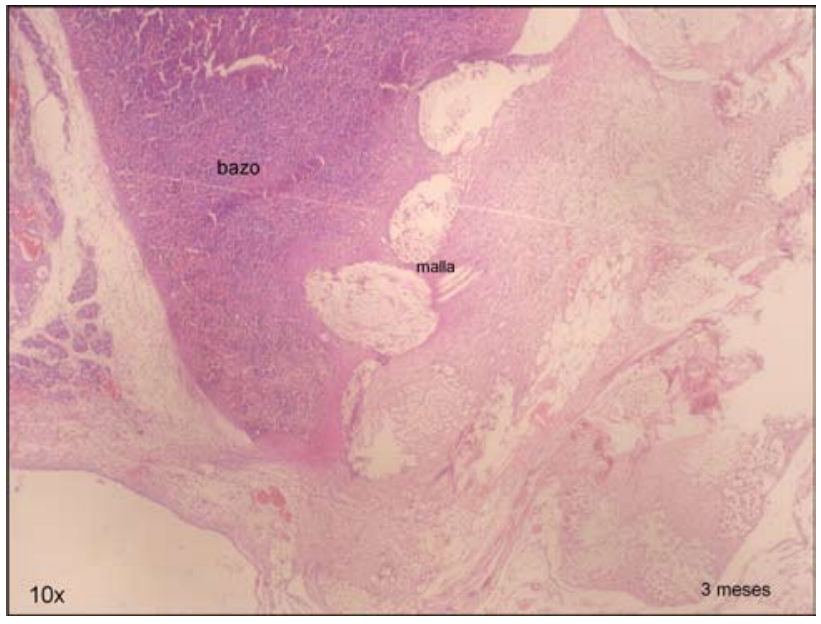

Figura 5. Coalescencia de la malla con el bazo.

reabsorvible podemos concluir que la conservación del bazo es el objetivo principal del trabajo, dado el alto costo (\$500 a $\$ 800)$ de la malla para nuestro medio, se espera que en un futuro no muy lejano sea posible el uso de la malla para preservar el bazo en casos de traumatismo, cohibir la hemorragia y evitar la exéresis $\left({ }^{9}\right)$.

\section{AGRADECIMIENTOS}

A la Dra. Melva Benavides Jefe del Servicio de Cirugía Experimental del Instituto Nacional de Salud del Niño, por permitir la culminación de este trabajo.

A Flor Cruz Chávez, Médica Veterinaria, por su paciencia y colaboración en el control pre y postoperatorio de los animales.

A Raquel Flores Salazar, Tecnóloga Médica.

A los trabajadores del Servicio de Cirugía Experimental de la Universidad Nacional Mayor de San Marcos y del Instituto Nacional de Salud del Niño, ex Hospital del Niño.

\section{REFERENCIAS BIBLIOGRÁFICAS}

1. Barrett J, Sheaff C, Abuabara S, Jonasson O. Splenic preservation in adults after blunt and penetrating trauma. Am J Surg. 1983;145:313-7.

2. Robbins. Pathologic Basis of Disease. 4th edition. Madrid: McGraw-Hill; 1990. p. 667-71. 
3. Robbins S. Structural and functional pathology. 4th ed. Mexico: Interamericana/McGraw-Hill; 1990.

4. Chryss C, Aaron WS. Successful treatment of rupture of normal spleen in newborn. Am J Dis Child. 1980;134:4189.

5. Beal SL, Spisso JM. The risk of splenorrhaphy. Arch Surg. 1988;123:1158-62.

6. Mieny CJ, Mennen U. Principles of surgical patient care. Abdominal Emergencies. Vol II Chapter IV. Pretoria: Academia, Johannesburg SA.; 1990. p. 51-2.

7. Singer DB. Post-splenectomy sepsis. In: Rosenberg HS, Bolande RP, eds. Perspectives in. pediatric pathology. Chicago: Year Book Medical Publishers, 1973. p. 285311.

8. Fasoli L, Bettili G, Bianchi S, Dal Moro A, Ottolenghi A. Spleen rupture in the newborn: conservative surgical treatment using absorbable mesh. J Trauma. 1998;45(3):642-3.
9. Delany HM, Porreca F, Mitsudo S, Solanki B, Rudavsky A.. Splenic capping: an experimental study of a new technique for splenorrhaphy using woven polyglycolic acid mesh. Ann Surg. 1982;196:187-93.

Manuscrito recibido el 22 de junio de 2007 y aceptado para publicación el 05 de setiembre de 2007.
Correspondencia:
Dra. Dina Sánchez Quispe
Facultad de Medicina - UNMSM
Av. Grau 755
Lima 1, Perú
Correo-e:mdisanchez@yahoo.com 